\title{
Le concept de champ pour une approche interdisciplinaire du tourisme
}

Discussion théorique à propos de Clivaz, Nahrath et Stock (2011), et perspectives de généralisation

André Suchet

\section{(2) OpenEdition}

\section{Journals}

Édition électronique

URL : http://journals.openedition.org/tourisme/74

DOI : $10.4000 /$ tourisme.74

ISSN : 2492-7503

Éditeur

Éditions touristiques européennes

Édition imprimée

Date de publication : 1 décembre 2013

Pagination : 2-10

ISSN : 2109-5671

Référence électronique

André Suchet, "Le concept de champ pour une approche interdisciplinaire du tourisme », Mondes du Tourisme [En ligne], 8 | 2013, mis en ligne le 01 juin 2015, consulté le 10 décembre 2020. URL : http:// journals.openedition.org/tourisme/74 ; DOI : https://doi.org/10.4000/tourisme.74

\section{c) (i)}

Mondes du tourisme est mis à disposition selon les termes de la licence Creative Commons Attribution - Pas d'Utilisation Commerciale - Pas de Modification 4.0 International. 


\title{
Le concept de champ pour une approche interdisciplinaire du tourisme Discussion théorique à propos de Clivaz, Nahrath et Stock (2011), et perspectives de généralisation
}

\author{
ANDRÉ SUCHET \\ [a.suchet@wanadoo.fr] \\ Docteur en géographie \\ Ingénieur de recherche CNRS (contractuel), laboratoire PACTE (UMR 5 194), Université de Grenoble/CNRS
}

Résumé. Cet article discute les apports et les limites d'un travail de Clivaz, Nahrath et Stock (20 I I), dont l'intention est de conceptualiser puis de caractériser l'existence d'un champ touristique mondial (en référence au concept sociologique de Bourdieu, 1984b, 1992). Par suite, l'article formule des éléments complémentaires de mise en œuvre et de généralisation d'une approche en termes de champ pour étudier le tourisme. Selon nous, il convient notamment de ne pas réduire au singulier ce concept de champ touristique (d'autres champs ou sous-champs existent indissociablement) ; la pluralité des situations mérite le pluriel conceptuel à plusieurs niveaux, y compris lorsque ces niveaux sont emboîtés les uns dans les autres.

Abstract. This article discusses the benefits and limits of the work of Clivaz, Nahrath and Stock (201 I), whose intention is to conceptualize and characterize the existence of a global tourism field (referring to the sociological concept of Bourdieu, 1984b, 1992). After this discussion, the article presents further elements in order to study tourism with the concept of field. In particular, we believe there is not only one tourism field, but the several existing situations require many conceptual levels, including when these levels overlap more or less each other. 
$\mathbf{P}$ our schématiser l'effet de concurrence entre les sites, les stations, ou plus largement les destinations touristiques et sportives, à partir de leur produit d'appel (le ski, le thermalisme, les activités de mer ou de montagne...), plusieurs études universitaires mobilisent la notion de “champ", plus ou moins référée à l'approche théorique de Bourdieu (1984b, 1992) en sociologie. On pense notamment aux travaux de Dienot et Theiller (1992), Bourdeau (2000), Keerle (2002) ou encore Hallé (2008). Plus précisément, on peut noter que les auteurs soit mobilisent de façon caricaturale le dispositif théorique de Bourdieu - c'est le cas de Hallé (2008), bien que le titre de sa recherche ne l'annonce pas -, soit sollicitent imperceptiblement les capacités du concept de champ, mais restent tellement prudents qu'ils ne citent pas Bourdieu ou s'en acquittent trop rapidement c'est le cas de Dienot et Theiller (1992) ; Bourdeau (2000). Plus encore, on pourrait surtout citer nombre de mémoires de recherche qui mobilisent sans aucune précaution le concept de "champ touristique" ou de "champ du tourisme". À la suite de ces études relativement démunies sur un plan théorique, un groupe d'auteurs basé à l'Institut universitaire Kurt Bösch de Sion formalise remarquablement le concept de champ pour des lieux géographiques mis en tourisme (Clivaz, Nahrath et Stock, 20। I). Ils affirment : "Nous appelons champ touristique cette configuration où la reconnaissance d'intérêts spécifiques permet aux acteurs des stations touristiques de mobiliser et de jouer leur capital [...] afin de se positionner avec leurs avantages concurrentiels dans ce jeu à la fois marchand et non marchand. [...] L'existence d'un champ touristique, où les agents seraient constitués par les acteurs des stations touristiques (voire par les stations en tant qu'acteurs collectifs)" (Clivaz, Nahrath et Stock, 2011, pp. 278-279). Bien qu'il s'agisse d'un modèle annoncé comme encore provisoire, cette publication de l'Institut universitaire Kurt Bösch dans la revue francophone de recherche Mondes $d u$ tourisme $^{(1)}$ ainsi que le programme collectif dont elle est tirée (Stock, 20।3) ${ }^{(2)}$, représentent, selon nous, une avancée majeure dans la recherche sur le tourisme.

Le présent article - après un cadrage disciplinaire, et en l'occurrence interdisciplinaire -, discute les apports et les limites de cette publication et formule certains éléments complémentaires de mise en œuvre et de généralisation d'une approche en termes de champ pour étudier le tourisme afin d'en arriver à une conceptualisation efficiente des effets de définition ou de redéfinition, de capitalisation ou de hiérarchisation, et surtout de concurrence relative entre les entités qui composent ce phénomène à travers le monde $^{(3)}$.

\section{UN TRAVAIL DE CONCEPTUALISATION INTERDISCIPLINAIRE}

Ce travail de conceptualisation, tout comme l'article de Clivaz, Nahrath et Stock (20II) auquel il apporte une suite et une réponse, est fondamentalement interdisciplinaire. Ainsi que le recommandent justement Darbellay et Stock (20 I 2a, 2012b) dans plusieurs articles du même programme de recherche, il s'agit de croiser la géographie, la sociologie, l'histoire, les sciences politiques (au pluriel comme au singulier) ou encore les sciences de gestion (elles-mêmes pluridisciplinaires), mais sans pour autant faire disparaître toute trace de rattachements disciplinaires (Darbellay et Paulsen, 2008 ; Rhein, 2003). Conséquemment, ce travail n'a aucune ambition transdisciplinaire - qui effacerait les frontières entre géographie, histoire, sociologie et psychologie ou science politique - et ne s'engage pas non plus dans la complexité d'une métadisciplinarité (cf. tableau 1).

Plus précisément, ce travail est interdisciplinaire dans la mesure où un apport de la sociologie modifie le raisonnement géographique, ou encore lorsque la géographie permet de lier des concepts de sociologie ou de science politique habituellement opposés par leur école de pensée (holisme/individualisme, structure/action, espace/territoire...). Par exemple, la notion de "champ du tourisme de montagne" permet de lier certains acquis de Bourdieu (1979, 1984b) avec l'étude des orga- 


\section{Tableau | • Définitions épistémologiques des pratiques de recherche entre plusieurs disciplines}

(adaptées de Darbellay et Paulsen, 2008 ; Béchillon, 1997 ; et Morin, 1994,

en ce qui concerne la métadisciplinarité)

Métadisciplinarité

Travail de recherche, à partir d'une écologisation des disciplines, c'est-à-dire en dépassant la segmentation tout en conservant les disciplines.

Transdisciplinarité(*) Processus de connaissance qui va au-delà des frontières disciplinaires. Affranchissement, reconfiguration ou disparition des partages disciplinaires dans une perspective systémique, globale et intégrée.

Appréhender le sujet dans sa complexité globale.

Interdisciplinarité

Mettre en intégration dynamique deux ou plusieurs disciplines pour décrire, analyser et comprendre un objet donné. Collaboration et intégration théorique entre les disciplines.

Enrichissement théorique mutuel entre spécialités et disciplines.

II peut s'agir d'opérations de transfert ou d'emprunts de concept ou de méthodes entre disciplines, voire la création de nouveaux champs de recherche par couplage de deux ou plusieurs disciplines, par exemple la géographie historique.

Pluridisciplinarité

(ou multidisciplinarité)
Un objet d'étude donné est abordé de manière successive ou juxtaposée par deux ou plusieurs points de vue disciplinaires sans véritable intégration entre ces bases disciplinaires.

Chaque contribution conserve la spécificité des concepts et méthodes de sa discipline.

But commun, travail par addition des apports ou division en sous-problèmes. Utiliser la complémentarité des disciplines pour la résolution d'un problème.

(*) Plusieurs auteurs, notamment au plan international, tiennent pour transdisciplinaire un processus de recherche qui intègre dans sa propre réalisation les acteurs politiques, sociaux, économiques et citoyens; un processus où les acteurs externes au domaine scientifique sont amenés à participer activement à la co-construction des connaissances (Hirsch Hadorn et al., 2008 ; Klein, 1990). Mais ce n'est pas la définition que nous accordons à "transdisciplinarité". Selon nous, ces questions relèvent davantage de la posture de recherche (avec les notions de "rechercheaction", "recherche appliquée" ou "recherche impliquée").

nisations locales d'une vallée (au sens de Crozier et Friedberg, 1977 ; ou de Friedberg, 1997). Dans ce cadre, l'objet même d'une étude faite à partir du concept de champ touristique ou de champ du tourisme n'est plus seulement géographique, ni seulement historique ou sociologique, " $i$ est co-construit dans et par le processus interdisciplinaire sur la base des compétences existantes, tout en étant irréductible à l'un ou l'autre des points de vue disciplinaires concernés” (Darbellay et Paulsen, 2008, p. 4).

\section{LE CONCEPT DE CHAMP POUR ÉTUDIER LE TOURISME}

Pour le dire de but en blanc, un des apports déterminants du travail de Clivaz, Nahrath et Stock (20I I) réside dans la démonstration de l'existence d'un champ spatialisé que représente la concurrence des stations entre elles. Conformément donc au principe de travail interdisciplinaire, qui ne signifie pas la disparition des rattachements disciplinaires en eux-mêmes, le concept de champ passe ainsi de la sociologie à la géographie. Comme le précisent Clivaz, Nahrath et Stock (201 I, pp. 278-279), il s'agit de la transposition des concepts de Bourdieu à des lieux géographiques. 
De plus, les auteurs de l'Institut universitaire Kurt Bösch se préoccupent de la concurrence des stations entre elles, mais leur démarche peut s'élargir, selon nous, à une destination ou, inversement, à tout espace socialement approprié par un groupe d'acteurs dont il est le support identitaire, économique et politique, c'est-à-dire une formation territoriale. Pour le dire simplement, on accorde une validité au principe de champ à d'autres échelles géographiques. Conceptuellement, même si le vocable de "territoire" n'est pas forcément sollicité, notamment au profit d'un vocabulaire spécifique au monde du tourisme (sites, stations, destinations...), ces dénominations correspondent à autant de lieux, de territoires de projet ou de "territoires qui se projettent" (Hazebroucq, 2009; Marchal et Duchesne, 2006). Sans pour autant formuler de correspondance entre site et lieu ou entre destination touristique et territoire, les dénominations par objets géographiques recoupent les concepts fondamentaux de lieu ou de territoire au sens de la géographie sociale (Raffestin, 1986 ; Berdoulay et Entrikin, 1998 ; Di Méo, 1998, 200I ; Giraut, 2008). Autrement dit, la concurrence au sein du champ se situe entre les sites touristiques, entre les stations ou entre les destinations, tout comme elle existe entre les lieux ou entre les territoires.

Enfin, sur le plan méthodologique, la crainte que manifestent Clivaz, Nahrath et Stock (20I I, p. 279) au sujet de la difficulté d'analyse induite par une délimitation floue du tourisme et des sous-champs de ce métasecteur ne nous semble pas un obstacle dans la mesure où les autres domaines étudiés par Bourdieu sont eux-mêmes variablement délimités. On pense notamment au champ littéraire, au champ religieux, au champ universitaire (Bourdieu, 197I, 199I, 1984a). Effectivement, Bourdieu (1992) estime que "les limites $d u$ champ sont elles-mêmes un objet de lutte au sein du champ" dans la mesure où la définition de ceux qui appartiennent ou non au champ pose justement la question "de la définition légitime de la pratique" ou de "la bonne manière de pratiquer". Autant de préoccupations fondamentales des occupants du champ.

Théoriquement, rappelons que Bourdieu (1984b, 1992) affirme la présence de champs, au sens de milieux spécifiques, résultant du processus historique de différenciation que connaissent les sociétés contemporaines et caractérisés par certaines propriétés. Bourdieu (1992, p. 73) note ainsi que "le cosmos social est constitué de l'ensemble de ces microcosmes sociaux relativement autonomes". Mais tout groupe de personnes ne constitue pas forcément un champ. Le concept de champ formulé par Bourdieu est un espace de lutte, "un réseau ou une configuration de relations objectives entre des positions". Ces positions sont définies objectivement dans leur existence et dans les déterminations qu'elles imposent à leurs occupants dans la distribution des différents capitaux en jeu dans le champ. Autrement dit, un champ se définit principalement par :

- La présence de capitaux spécifiques ou non. L'argent, le capital social (les relations entre personnes), les diplômes et certificats sont des capitaux non spécifiques, c'est-àdire actifs dans l'ensemble de la société. Le nombre de lits d'une vallée en Haute-Savoie est un capital spécifique au tourisme. De même, un champion du monde de patinage artistique représente pour une destination de montagne un capital très spécifique pour atteindre notamment la clientèle féminine ou senior. Autant d'aspects que les auteurs de l'Institut universitaire Kurt Bösch tentent de formaliser par le concept intégratif de "capital touristique" (Darbellay, Clivaz, Nahrath et Stock, 20I I). Cette nouvelle formalisation paraît stimulante mais encore très délicate à utiliser. On ne s'engage pas ici sur cet aspect. Les chaînes hôtelières disposent d'un certain nombre de lits afin de loger un maximum de personnes, mais, à partir des bénéfices, il s'agit encore d'augmenter la capacité d'hébergement. De même, l'accumulation de devises étrangères en station de sports d'hiver est une fin, mais elle est aussi un moyen de croissance par capacité d'investissement, puis un moyen de dominer le champ international des destinations hivernales.

- La présence d'une certaine croyance en la valeur des capitaux, en l'importance du domaine, et plus largement la présence d'un intérêt au jeu du champ : ce que Bourdieu 
nomme en latin l'illusio. Pour le dire très simplement : "Les individus doivent y croire." Par exemple, certaines nations d'Asie centrale ou d'Afrique du Nord ne souhaitent pas s'engager dans une mise en tourisme à laquelle ils attribuent des valeurs négatives (occidentalisation, capitalisme, perte des traditions...) et restent à l'écart du champ, alors même que beaucoup d'autres États pensent leur avenir dans le tourisme. Ces derniers témoignent d'un illusio en faveur du tourisme et de ses capitaux (nombre d'arrivées, devises étrangères...).

- La présence d'une lutte interne pour sa domination. Une concurrence objective qui passe par une concurrence subjective pour l'imposition des règles de hiérarchisation, c'est-à-dire tout à la fois les limites du champ (les concurrents admis) et la valeur des capitaux. Dans un champ, les personnes investissent des stratégies de conservation (maintenir la structure du champ) ou des stratégies de subversion (réorganiser le champ à son avantage). Dans le domaine sportif longuement étudié par quelques-uns des étudiants de Bourdieu - et par lui-même à plusieurs reprises -, cette lutte correspond à une lutte pour la définition légitime de la pratique (Bourdieu, 1987, pp. 203-216, 1992 ; Clément, 1994). Par exemple, en escalade, durant les années 1980, les partisans de la compétition tentent d'imposer l'escalade sportive, contre les tenants d'un alpinispe classique. Chacun des partis rivalise d'arguments pour imposer sa définition légitime de l'activité dans le champ, c'est-à-dire aussi la hiérarchie des légitimités entre les différentes formes de pratique. Comme l'attestent les études de Pociello (1995) et celles qu'il a dirigées à l'université d'Orsay, le même mécanisme se retrouve en nautisme, en rugby, en vol libre et dans bien d'autres activités.

Il en est rigoureusement de même pour les destinations touristiques. Les stations, les sites pittoresques aménagés ou les zones urbanisées sont en concurrence, et cherchent à se positionner au sein d'un champ touristique ou sportif (Clivaz, Nahrath et Stock, 20 I I). Parmi les stations de sports d'hiver, chacune tente d'imposer la longueur de piste, la qualité environnementale, le nombre de remontées mécaniques ou autres critères avantageux pour s'afficher comme étant "la plus grande station de ski", "le plus grand domaine skiable d'Europe", "la plus belle station"... Effectivement, nul meilleur moyen n'existe pour être classé premier que de formuler soimême les critères du classement en ayant déterminé les candidats autorisés à concourir. Plus encore : villégiature, ascensionnisme et séjours en montagne, sports d'hiver, thermalisme, climatisme, thalassothérapie, tourisme balnéaire, tourisme d'aventure... sont autant de catégories subjectives différenciées au cours du temps (Boyer, 1996 ; Équipe Mit, 2002, 2005 ; Knafou et Violier, 2000 ; Réau, 2007 ; Bataillou, 2010 ; Cousin et Réau, 2009), c'est-à-dire autant de champs ou de souschamps dans lesquels une station, une vallée ou toute autre destination tente d'imposer sa prééminence ( $c f$. figure 1).

Finalement, si l'apport essentiel des auteurs de Sion est de formaliser la dimension spatiale du tourisme en tant que champ économiquement, socialement, politiquement et symboliquement constitué, séparer la concurrence en jeu dans l'espace de celle en jeu dans l'ensemble de la société ne semble pas satisfaisant. Considérant l'influence du non-spatial sur le spatial, et inversement, à tous les niveaux (Frémont, Chevalier, Hérin et Renard, 1984 ; Chadefaud, 1988 ; Di Méo, 1998 ; Séchet et Veschambre, 2006 ; Di Méo et Buléon, 2005), voire même la concurrence entre objets spatiaux et non spatiaux (la limitation du temps disponible impose un choix entre la lecture, la télévision, les voyages ou le travail), on gardera l'idée d'un ensemble imbriqué dans d'autres ensembles (le sport, les loisirs, le travail...). Ainsi, plutôt que de se réduire formellement à ce champ touristique spatialisé que pointent Clivaz, Nahrath et Stock (20I I), on estime - tout en reconnaissant l'existence de ce champ - qu'il faut étudier l'ensemble du jeu de concurrence entre les lieux, mais aussi entre acteurs et entre institutions situés hors de ces lieux (les maisons d'édition qui publient des livres de voyages en faveur de l'une ou l'autre des destinations, mais aussi les dirigeants de salles de cinéma qui représentent un groupe d'acteurs concurrents). Non pas que le champ touristique au sens de Clivaz, Nahrath et Stock (20I I) 


\section{Figure | - Quelques-uns des champs et des sous-champs du tourisme}

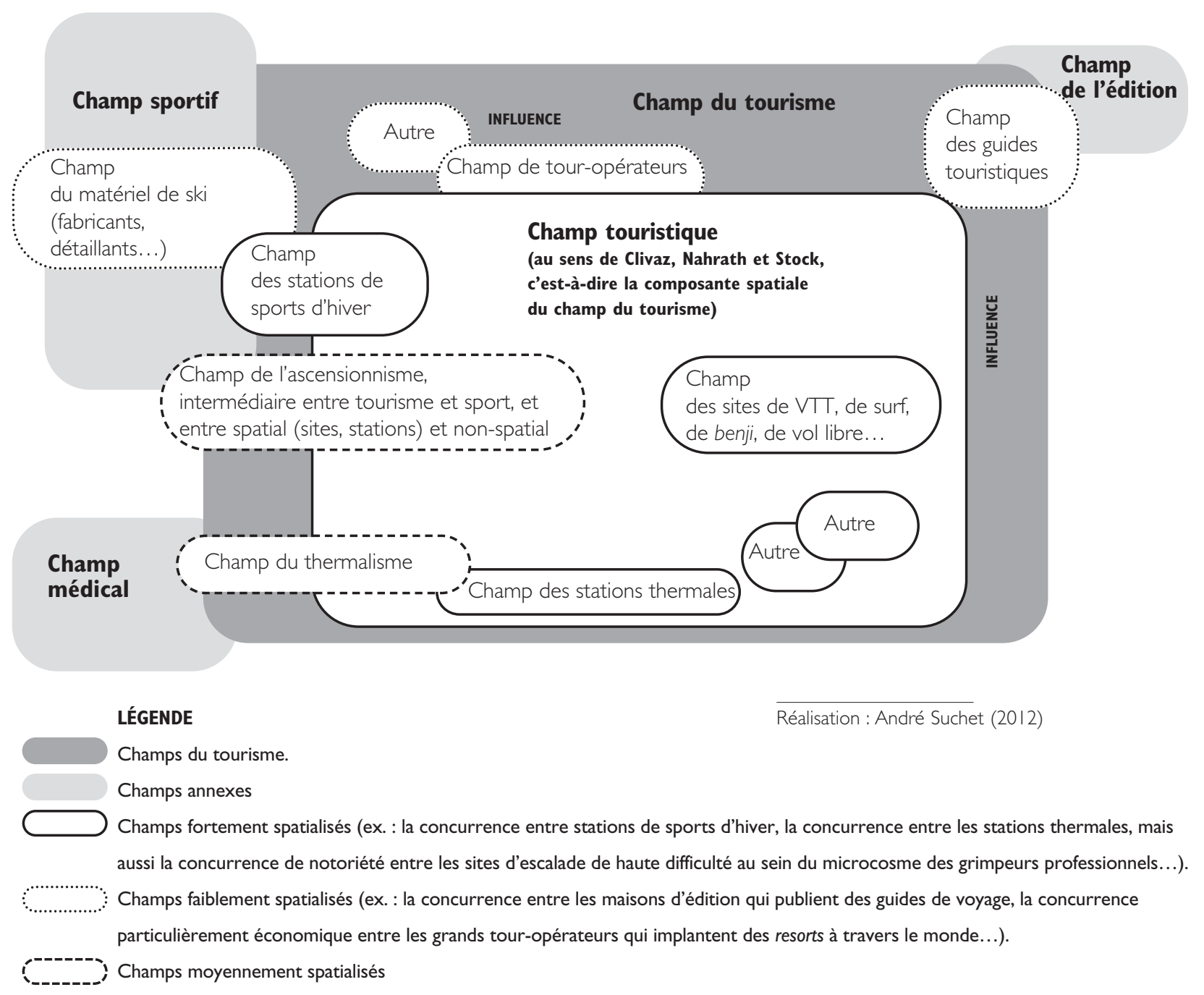

N.B. : Les rapports entre sport et loisirs ou entre tourisme et loisirs ne sont pas détaillés ici (voir à ce sujet Sue, 1993 ; Brunet, 1997 ;

Terret, 2003).

ne soit pas un des secteurs importants du phénomène touristique mondial, mais dans le sens où il n'est que l'une des pièces d'un mécanisme global qu'il convient de ne pas trop longuement isoler au cours d'une recherche. On recommande donc d'utiliser ce concept de champ touristique, mais aussi et tout autant celui légèrement plus large de champ du tourisme, ou inversement celui plus restreint de sous-champ des stations de sports d'hiver (évidemment sans écrire nécessairement chaque fois le préfixe "sous"), et toutes les combinaisons possibles selon la centration du raisonnement sur l'un ou l'autre des aspects que l'on souhaite appréhender (le champ ou souschamp des destinations thermales, le champ du tourisme culturel, le champ du tourisme souterrain, le champ des sites d'art roman, le champ des parcs à thème, etc.), tout en reconnaissant la pluralité des relations sociales et spatiales en jeu et en examinant les relations de ce champ - momentanément isolé pour les besoins de la recherche - avec un ou plusieurs autres champs selon 
le cas d'étude - le "champ du pouvoir", comme le recommandait Bourdieu (1987, 199 |), lorsque cela paraît pertinent, mais aussi le champ sportif ou encore le champ médical.

\section{CONCLUSION}

À la suite de multiples études relativement démunies sur un plan théorique, l'équipe universitaire de l'Institut Kurt Bösch en Suisse formalise remarquablement le concept de champ pour des lieux géographiques mis en tourisme. Sur la base du travail sociologique de Bourdieu (1984b, 1992), le texte rédigé par Clivaz, Nahrath et Stock (20I I) ainsi que le programme collectif dont il est tiré (Stock, 2013) représentent, selon nous, une avancée majeure dans la recherche en tourisme.

Plus précisément, un des apports déterminants du travail de Clivaz, Nahrath et Stock (20I I) réside dans la démonstration interdisciplinaire de l'existence d'un champ spatialisé que représente la concurrence des stations entre elles et que l'on peut admettre à d'autres échelles géographiques (y compris à l'échelle conceptuelle du lieu ou du territoire). Les limites de cette "première brique” empêchent cependant une généralisation solide. Séparer la concurrence en jeu dans l'espace de celle en jeu dans l'ensemble de la société ne nous semble pas satisfaisant, et l'on considère plutôt avec les acquis déjà anciens de la géographie sociale qu'il existe une influence du non-spatial sur le spatial, et inversement, mais aussi une concurrence entre objets spatiaux et non spatiaux. Selon nous, il convient donc de ne pas réduire au singulier ce concept de champ touristique (d'autres champs ou souschamps existent indissociablement) : la pluralité des situations mérite indispensablement le pluriel conceptuel à plusieurs niveaux, y compris lorsque ces niveaux sont emboîtés les uns dans les autres. Ce plurielqui consiste à se focaliser sur l'un ou l'autre des champs et des souschamps du tourisme, tout en étudiant les relations de ce dernier avec d'autres champs situés hors du tourisme - permet, dès lors, une généralisation à la fois simple et rigoureuse.

-
Notes

(*) Remerciements à loan Chioar pour ses conseils avisés au moment de l'écriture de ce texte.

( I) L'article qui nous préoccupe figure dans un numéro hors-série de cette revue, correspondant aux actes des III Res Rendez-vous Samuel Champlain du tourisme co-organisés en 2010 par l'Université d'Angers:

Christophe Clivaz, Stéphane Nahrath et Mathis Stock, "Le développement des stations touristiques dans le champ touristique mondial", Mondes du tourisme, hors-série

“Tourisme et mondialisation”, 201 I.

(2) Pour d'autres résultats théoriques ou empiriques de ce groupe de recherche, voir notamment Clivaz, Nahrath et Stock (20I0), Stock (20I0), ou encore Nahrath et Stock (20|2).

(3) Cette publication de recherche théorique et de réponse aux travaux de l'équipe universitaire de Sion repose initialement sur un travail de thèse en géographie du tourisme (Suchet, 2012). 


\section{CHRONIQUE SCIENTIFIQUE}

Pierre Bourdieu, Homo academicus, Minuit, 1984a.

Pierre Bourdieu, Questions de sociologie, Minuit, 1984b.

Pierre Bourdieu, Choses dites, Minuit, 1987.

Pierre BOURDIEU, "Le champ littéraire", Actes de la recherche en sciences sociales, $n^{\circ} 89,1991$.

Pierre Bourdieu, "La logique des champs", dans Pierre Bourdieu et Loïc J. D. WACQuANT (dir.), Réponses. Pour une anthrophologie réflexive, Seuil, 1992.

Marc BOYER, L'Invention du tourisme, Gallimard, 1996.

Roger BRUNET, "Les mots de la géographie : tourisme et loisirs", L'Espace géographique, vol. 26, n 3, 1997.

Michel Chadefaud, Aux origines du tourisme dans les pays de l'Adour. Du mythe à l'espace : un essai de géographie historique, J\&D éditions (thèse de doctorat d'État en géographie, publiée post-mortem), 1988. Jean-Paul ClÉMENT, "Les apports de la sociologie de Pierre Bourdieu à la sociologie des sports", revue Staps, vol. 15, n 35, 1994. Christophe Clivaz, Stéphane NaHrath et Mathis Stock, "Le développement des stations touristiques dans le champ touristique mondial", Mondes du tourisme, hors-série "Tourisme et mondialisation", 201 I.

Christophe Clivaz, Stéphane NaHrath et Mathis Stock (dir.), "Tourisme, urbanité, durabilité", dossier thématique de Urbia. Les Cahiers du développement urbain durable, $n^{\circ} 10,2010$.

Saskia Cousin et Bertrand RÉAu, Sociologie du tourisme, La Découverte, 2009.

Michel Crozier et Erhard Friedberg, L'Acteur et le Système, Seuil, 1977.

Frédéric Darbellay, Christophe Clivaz, Stéphane Nahrath et Mathis SтосK, "Approche interdisciplinaire du développement des stations touristiques. Le capital touristique comme concept opératoire", Mondes du tourisme, $\mathrm{n}^{\circ} 4,201 \mathrm{I}$.

Frédéric Darbellay et Theres PAULSEN, Le Défi de l'inter- et transdisciplinarité. Concepts, méthodes et pratiques innovantes dans l'enseignement et la recherche, Presses polytechniques et universitaires romandes, 2008.

Frédéric DarbelLaY et Mathis Stock, "Penser le touristique : nouveau paradigme ou interdisciplinarité ?", EspacesTemps.net, 20 I2a [en ligne, à partir de http://uww.espacestemps.net/articles/penser-le-touristique-nouveau-paradigme-ou-interdisciplinarite].
Frédéric Darbellay et Mathis Stock, "Tourism as complex interdisciplinary research object", Annals of Tourism Research, vol. $30, n^{\circ} 1,2012 b$

Guy Dı Méo, Géographie sociale et territoires, Nathan, 1998. Guy Dı MÉo, "De l'effet de lieu au territoire : la question du sujet et de la territorialité", dans Jean-Marc Fournier (dir.), Faire la géographie sociale aujourd'hui, Presses universitaires de Caen, 2001.

Guy Dı MÉo et Pascal BulÉon (dir.), L'Espace social. Une lecture géographique des sociétés, Armand Colin, 2005.

Josy DienOt et Didier Theiller, "Les nouvelles pratiques sportives de la montagne: un champ nouveau pour le tourisme de pleine nature", dans Tourisme et environnement, La Documentation française, 1992.

ÉQUIPE MIT, Tourismes I : Lieux communs, Belin, 2002.

ÉQUIPE MIT, Tourismes 2: Moments de lieux, Belin, 2005.

Armand Frémont, Jacques CheValier, Robert HérIN et Jean

Renard, Géographie sociale, Masson, 1984.

Erhard Friedberg, Le Pouvoir et la Règle. Dynamique de l'action organisée, Seuil, 1997.

Frédéric GIRAUT, "Conceptualiser le territoire", Historiens et géographes, n 403, 2008.

Julie Hallé, L'État de l'organisation du tourisme sportif en Asie centrale (Kazakhstan, Kirghizstan, Ouzbékistan, Tadjikistan). Acteurs et structures dans un modèle en transition, thèse de doctorat, Université Grenoble I, 2008.

Jean-Marie HazebroucQ, "De l'espace touristique à la 'destination touristique'. Un 'territoire' qui se projette", dans Jean-Pierre Lemasson et Philippe VIOLIeR (dir.), Destinations et territoires, Presses de I'Université du Québec, 2009.

\section{Gertrude Hirsch Hadorn, Holger HoffmanN-Riem, Susette} Biber-Klemm, Walter Grossenbacher-Mansuy, Dominique

JOYE, Christian POHL et al. (dir.), Handbook of Transdisciplinary Research, Springer, 2008.

Régis Keerle, Sports et territoires. Contribution à une géographie du pouvoir : une géographie sociale du champ sportif, thèse de doctorat, Université Montpellier III, 2002.

Julie Thompson KLeIN, Interdisciplinarity: History, Theory, and Practice, Wayne State University Press, 1990. 
Rémy KNAFOU et Philippe VIOLIER, "Tourismes en France : vivre de la diversité", Historiens et géographes, $n^{\circ} 370,2000$.

Christophe MARChal et Arnaud DUCHESNe, "Territoire de projet et destination touristique : des notions à clarifier", Intercommunalité et tourisme, "Cahier Espaces", n 91, 2006.

Edgar MORIN, De l'interdisciplinarité", dans François KOURILSKY (dir.), Interdisciplinarité, CNRS Éditions, 1994.

Stéphane NAHRATH et Mathis Stock (dir.), Urbanité et tourisme, numéro thématique de Espaces et sociétés, $n^{\circ}$ 151, 2012.

Christian Pociello, Les Cultures sportives, Presses universitaires de France, 1995.

Claude RAFFESTIN, "Nature et culture du lieu touristique",

Méditerranée, vol. 58, nº 3, 1986.

Bertrand RÉAU (dir.), "Nouvelles (?) frontières du tourisme", numéro thématique de Actes de la recherche en sciences sociales, $n^{\circ} 170$,

2007.

Catherine RHein, L'espace, les sociologues et les géographes.

Déconstruire et reconstruire les 'disciplines' : les jeux de l'interdisciplinarité", Sociétés contemporaines, n 49-50, 2003.

Raymonde SÉCHET et Vincent VESCHAMBRE (dir.), Penser et faire la géographie sociale. Contributions à une épistémologie de la géographie sociale, Presses universitaires de Rennes, 2006.

André SUCHET, Acteurs, gouvernance et dynamiques de projet dans la concurrence des territoires en tourisme. Étude autour du programme sportif Pyrénées : Laboratoire de l'Olympisme 1988-1994 et des Jeux Pyrénéens de l'Aventure 1993, thèse de doctorat en géographie, université de Grenoble, 2012.

Mathis STOCK (dir.), "Vers une théorisation de l'approche géographique du tourisme", dossier thématique de Mondes du tourisme, $\mathrm{n}^{\circ} 2,2010$.

Mathis Sтоск (dir.), Entre abîme et métamorphose. Une approche interdisciplinaire du développement des stations touristiques, recherche en cours financée par le Fonds national suisse de la recherche scientifique et le canton du Valais, 20 I - 20 I4.

Roger SUE, Le Loisir, Presses universitaires de France, 1993.

Thierry TeRRet (dir.), Éducation physique, sport et loisir 1970-2000,

AFRAPS, 2003. 